\title{
Conformity of Fruit Nectar Samples to Libyan Specification Standards
}

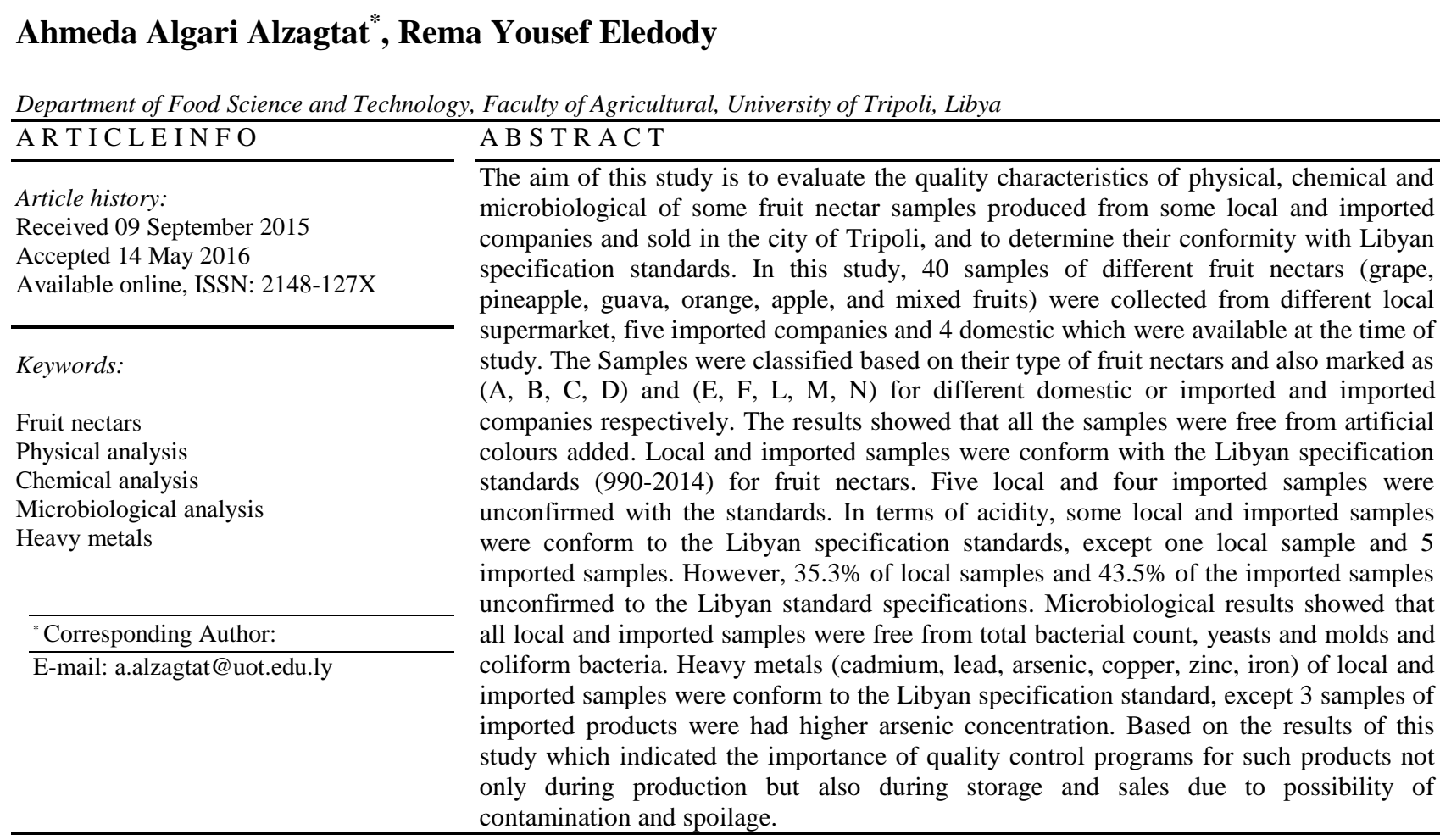

\section{Introduction}

Nectars, juices and drinks produced from fruits are the most common industries, which are prevalent in most parts of the world, global demand for such products has increased over recent decades. These industries are depend on some raw materials such as fruits concentrate, sugar and additives (natural flavors and sweeteners) which are permitted for use. The most important factors that determine the type of product (juice, nectar or drink) are the ratio of natural juice or natural concentrate used for production, generally, the proportion of natural juice in the juice product should be more than $50 \%$ and nectars should be between $25-50 \%$ and drinks should be between 10-24\% depending on the type of fruit (Codex Alimentarius, 2005).

Fruit nectar is the unfermented but fermentable product obtained by adding water with or without the addition of sugars, honey and/or syrups, and/or permitted food additive sweeteners to products or to a mixture of those products. Aromatic substances, volatile flavor components, pulp and cells all of which must be recovered from the same kind of fruit and be obtained by suitable physical means may be added, that product moreover must meet the requirements defined for fruit nectars standard, a mixed fruit nectar is obtained from two or more different kinds of fruits (NCSM, 2014).

Nectars and juices contain some good nutrients such as natural sugars and certain vitamins and minerals, they are also of refreshing products and desired by the consumer, especially children. Consumption of these products depends on food pattern, and economic terms of the individual and family (ASSABAH, 2014). Some producers when producing such products may be used some additives which are not generally recognized as safe (GRAS) additives such as some pigments or colors and preservatives which may be carcinogenic, so legislative and specifications bodies approved several technical and health requirements, therefore, quality control programs and testing for such products is important in order to prevent consumer health and safety.

Some researchers in Romanian (2013) conducted a study, and the aim of this study was to analyze changes for physicochemical properties ( $\mathrm{pH}$ values, acidity, vitamin $\mathrm{C}$, and total soluble solids) of fresh and refrigerated stored of fruit juices for a week. Fruit juices (orange, kiwi, apple and mixed of different fruits) were prepared and study their physicochemical characterization. Results showed that kiwi juice and 
mixed kiwi and orange 30:70\% contained a high amount of vitamin C, (83.6 and $960.8 \mathrm{mg} / 100 \mathrm{~g})$ respectively. As it turns out that the reduction in the amount of vitamin $\mathrm{C}$ per $100 \mathrm{~g}$ of the product to be clear in orange juice and mixed juice after the storage process, also there were no significant differences in the other physicochemical properties for fresh and stored juices (Leahu et al., 2013). A study in Zimbabwe (2013) was conducted to identify adulteration of fruit juices where 6 different varieties of fruit juices assembled, and the samples were analyzed and determining the quantity of heavy metals, acidity, total soluble solids (TSS \%) and inverted sugars. Results showed significant differences in the proportion of natural juice and added water in some samples, natural juice ratio were low (20.65\%), which refers to addition of high quantity of water, as well as the results showed that the proportion of TSS\% were low in $19.5 \%$ of the samples. The study also pointed out that the addition of sucrose was high in some samples which contrary to specification standards (Maireva et al., 2013).

A microbiological study of vendor and packed fruit juices locally available in Dhaka city Bangladesh was conducted in 2013. Twenty six different fruit juice samples handy or manually filled and fifteen factory processed fruit juices samples sold in the local market were analyzed. Results were revealed that, total bacterial count ranged between $10^{2}-10^{7}(\mathrm{cfu} / \mathrm{ml})$, Staphylococci bacteria present in thirty samples, the number of coliform bacteria ranged between $10^{2}-10^{6}(\mathrm{cfu} / \mathrm{ml})$, coliform bacteria was present in four samples of manually filled samples $\left(10^{2} \mathrm{cfu} / \mathrm{ml}\right)$, while the processed samples were showed absence of coliform bacteria. The study concluded that both types (manually filled and factory processed) fruit juices were low quality. Regulatory and authorities administrations must take quick action towards fruit juices factories and production units, regards health aspects in order to prevent safety and health of the consumers (Rashed et al., 2013).

A study was conducted (2004) in Brazil to determine copper and zinc concentration in fruit juice for 12 different sample. Results showed variation in the quantity of copper ranged between $85-384 \mathrm{mg} / \mathrm{kg}$ and the quantity of zinc ranging from the $68-1097 \mathrm{mg} / \mathrm{kg}$. The results indicated that all the samples were in permissible limits of standards for those two elements (Nascentes et al., 2004). A group of researchers in (2013) conducted a study to determine the concentration of some heavy metals in fruit juices and soft drinks available in the market of Accra city in Ghana. Twenty samples were bought from the market and the concentration of some heavy metals determined by atomic absorption spectrometer. Results showed that the average concentrations in fruit juice and soft drink samples were $0.83,9.07,1.59,3.33 \mathrm{mg} / \mathrm{kg}$ for copper, iron, lead and zinc respectively, also it showed that the concentration of heavy metals in some samples of fruit juices and soft drinks were higher than the permissible limits indicated by the World Health Organization (WHO) (Ofori et al., 2013).

As a result of different trade names, brands and varieties of locally produced and imported fruit nectars available in Libyan market, in addition to that, growing demand for consumption of different fruit juices and nectars by consumers, that highlighted the importance of conducting this study in order to evaluate physical, chemical and microbiological quality properties for different fruit nectar trade names, locally produced and imported and to evaluate their extent of compliance with the Libyan specification standard.

\section{Materials and Methods}

\section{Samples}

Forty samples were collected from local markets of the city of Tripoli area, which represent four local companies or producers and five foreign companies and 6 species varieties of different fruit nectars (pineapple, orange, guava, apple, grapes and mixed fruits).

Questionnaire included a series of questions was promoted in order to determine the per capita consumption of juices and nectars rate annually to compared with some other countries, also to know the most consumed brands and to determine the percentage of consumption of these products. More than 100 hard copy questionnaires were distributed to consumers in different areas of Libya, Also, e-questionnaire (electronic copy) was posted in the internet for Libyan consumers and 197 responses were obtained and then statistically and graphically analyzed.

\section{Methods of Analysis}

Physical and chemical methods: Water capacity test was conducted by measuring the volume of nectar in packages, then each package was filled with water to measures complete volume and water capacity was calculated according to the following equation:

Water capacity $\%=$ nectar volume/water volume $\mathrm{x} 100$

Total volume of nectar juices in the packages were also measured and compared with the volume written on the label of each package. Total titratable acidity was determined on the basis of malic acid for apple nectars, on the basis of tartaric acid for grape nectars and on the basis of citric acid for other nectar fruits according to (ISO, 1998b) method no. 750. Value of $\mathrm{pH}$ was measured accordance to (ISO, 1991) method no. 1842 by pH-meter. Total soluble solids (TSS \%) was determined according to (ISO, 1998a) method no. 2173, by refractmeter (ATAGOPR2011). Artificial colors test was conducted according to (IFU, 2005) method no. 24. Heavy metals were determined by (AOAC, 1984) method no. 25.061, using atomic absorption spectrophotometer.

Microbiological methods: Total bacterial count test was conducted by (IFU, 1996a) method no. 2 using a nutrient medium (Plant Count Agar) and incubated at $\left(37^{\circ} \mathrm{C}\right)$ for a period of $24-48$ hours. Coliform bacteria conducted by (ISO, 2006) methods no. 4832-1\&2 using nutrient medium (Hicrome ECC Selective Agar Base) and incubated at $\left(37^{\circ} \mathrm{C}\right)$ for $24-48$ hours. Yeasts and molds were conducted in accordance to (IFU, 1996b) methods 
no.3\&4 using nutrient medium (Chloramphenicol Yeast Glucose Agar) and incubated at $\left(25^{\circ} \mathrm{C}\right)$ for 3-5 days.

Readings and results of all the tests (physical, chemical and microbiological) were conducted in three replicates, and the results were subjected to statistical analysis using a computer with Minitab, ver. 14 program.

\section{Results}

\section{Water Capacity}

Water capacity value of the different local and imported fruit nectar containers were ranged from (89.90$95.23 \%$ ), which indicated that all the samples matching the Libyan specification standard no. (990-2014) for fruit nectars, as shown in tables ( $2 \& 3)$.

\section{Artificial Colors}

Tables ( 1 and 2) illustrated that all local and imported fruit nectars samples did not contain artificial colors added, so consequently all samples conform with the Libyan specification standard which mentioned above.

\section{$p H$}

Table 1 showed that local fruit nectar samples conform with the Libyan specification standard except the samples no. (4 pineapple, 5 pineapple, 8 mixed, 11 guava\&16 apple) for the subsidiaries (a, b, a, b, c) respectively. Table 2 showed that imported fruit nectar samples were also within Libyan specification standard, except 4 samples, namely as, (2 grape, 9 mixed, 14 guava, 17 orange) produced by (1, f. n. 1), because their $\mathrm{pH}$ values were higher than the limit of $\mathrm{pH}(3-4)$ which indicated in the Libyan specification standard (990-2014).

\section{Acidity}

Titratable acidity for all local fruit nectar samples were matching the Libyan specification standard (9902014) for fruit nectars, except one sample no. 9 (mixed) produced by the company (c) was higher than the limit, the highest acidity value for local samples was $(0.66 \%)$ for mixed fruit nectars no. 9, and the lowest value was $(0.22 \%)$ for grape sample no. 3 , as showed in Table 1. However, five samples of imported fruit nectar were not conformed to the specifications, namely, (1 grape, 3 pineapple, 4 pineapple, 5 pineapple, 21 apple), produced by (e, e, f, 1, f), companies respectively, the highest acidity value for imported samples was $(0.98 \%)$ for orange nectars no. 18 and the lowest value was $(0.12 \%)$ for orange sample no. 19 as indicated in Table 2, according to Libyan specification standard (990-2014), the acidity must not exceed $0.5 \%$ of different fruit nectars, except for orange nectars should be not more than $(1.0 \%)$.

\section{Total Soluble Solids (TSS\%)}

Table 1 indicated that TSS\% for some of the local fruit nectar samples ( 2 grape, 6 pineapple, 7 pineapple, 8 mixed, 9 mixed and 16 apple) were less than the limit in the Libyan specification standard, the highest TSS\% was $(16.1 \%)$ for both grape sample no. 3 and apple sample no. 17, and the lowest TSS\% were $5.5 \%$ for mixed sample 9 . At the meantime, Table 2 showed that TSS\% for some of imported samples numbered as ( 3 pineapple, 4 pineapple, 5 pineapple, 9 mixed, 10 mixed, 15 orange, 16 orange, 17 orange, 20 apple and 21 apple) were, also less than the limits showed in the Libyan specification standard for fruit nectars, and the highest TSS\% for was $15.2 \%$ for apple sample no.22, and the lowest TSS\% was $4.4 \%$ for mixed sample no. 10 .

Table 1 Physicochemical characteristics of local produced fruit nectars

\begin{tabular}{|c|c|c|c|c|c|c|c|c|}
\hline $\begin{array}{c}\text { Sample } \\
\text { No. }\end{array}$ & Product & $\begin{array}{c}\text { Company } \\
\text { Name }\end{array}$ & $\begin{array}{l}\text { Volume of } \\
\text { Nectars }\end{array}$ & $\begin{array}{c}\text { Water } \\
\text { Capacity \% }\end{array}$ & pH Value & Acidity \% & TSS \% & $\begin{array}{c}\text { Artificial } \\
\text { Colors }\end{array}$ \\
\hline 1 & Grape & $\mathrm{B}$ & $1000 \pm 10$ & $92.67 \pm 2.21$ & $3.68 \pm 0.11$ & $0.49 \pm 0.08$ & $15.5 \pm 0.5$ & -ve \\
\hline 2 & Grape & $\mathrm{C}$ & $1000 \pm 10$ & $93.10 \pm 3.11$ & $3.51 \pm 0.13$ & $0.32 \pm 0.06$ & $7.8 \pm 0.2$ & -ve \\
\hline 3 & Grape & $\mathrm{D}$ & $980 \pm 10$ & $91.16 \pm 1.34$ & $3.28 \pm 0.12$ & $0.22 \pm 0.05$ & $16.1 \pm 0.3$ & -ve \\
\hline 4 & Pineapple & A & $980 \pm 5$ & $89.90 \pm 1.23$ & $4.19 \pm 0.10$ & $0.49 \pm 0.06$ & $13.4 \pm 0.3$ & -ve \\
\hline 5 & Pineapple & B & $1000 \pm 15$ & $92.67 \pm 1.56$ & $4.89 \pm 0.25$ & $0.50 \pm 0.08$ & $15.1 \pm 0.3$ & -ve \\
\hline 6 & Pineapple & $\mathrm{C}$ & $1000 \pm 10$ & $93.10 \pm 2.21$ & $3.96 \pm 0.12$ & $0.45 \pm 0.06$ & $11.5 \pm 0.2$ & -ve \\
\hline 7 & Pineapple & $\mathrm{D}$ & $980 \pm 10$ & $91.16 \pm 1.15$ & $4.08 \pm 0.14$ & $0.33 \pm 0.05$ & $11.9 \pm 0.4$ & -ve \\
\hline 8 & Mixed & A & $980 \pm 10$ & $89.90 \pm 1.30$ & $4.13 \pm 0.10$ & $0.35 \pm 0.04$ & $13.0 \pm 0.3$ & -ve \\
\hline 9 & Mixed & $\mathrm{C}$ & $1000 \pm 12$ & $93.10 \pm 2.31$ & $4.01 \pm 0.09$ & $0.66 \pm 0.05$ & $5.5 \pm 0.2$ & -ve \\
\hline 10 & Guava & A & $980 \pm 10$ & $89.90 \pm 1.23$ & $4.00 \pm 0.08$ & $0.26 \pm 0.04$ & $12.8 \pm 0.4$ & -ve \\
\hline 11 & Guava & B & $1000 \pm 8$ & $92.67 \pm 1.97$ & $4.85 \pm 0.13$ & $0.51 \pm 0.08$ & $13.9 \pm 0.5$ & -ve \\
\hline 12 & Guava & $\mathrm{C}$ & $1000 \pm 10$ & $93.10 \pm 2.20$ & $4.04 \pm 0.08$ & $0.33 \pm 0.04$ & $12.6 \pm 0.3$ & -ve \\
\hline 13 & Orange & A & $980 \pm 10$ & $89.90 \pm 1.12$ & $4.00 \pm 0.11$ & $0.59 \pm 0.05$ & $12.6 \pm 0.4$ & -ve \\
\hline 14 & Orange & B & $1000 \pm 10$ & $92.67 \pm 1.75$ & $4.05 \pm 0.09$ & $0.65 \pm 0.07$ & $12.5 \pm 0.5$ & -ve \\
\hline 15 & Apple & A & $980 \pm 10$ & $89.90 \pm 1.31$ & $3.64 \pm 0.08$ & $038 \pm 0.08$ & $13.0 \pm 0.3$ & -ve \\
\hline 16 & Apple & $\mathrm{C}$ & $1000 \pm 20$ & $93.10 \pm 2.25$ & $4.99 \pm 0.13$ & $0.40 \pm 0.05$ & $10.7 \pm 0.2$ & -ve \\
\hline 17 & Apple & D & $980 \pm 10$ & $91.16 \pm 1.25$ & $3.89 \pm 0.09$ & $0.33 \pm 0.06$ & $16.1 \pm 0.5$ & -ve \\
\hline
\end{tabular}


Table 2 Physicochemical characteristics of imported fruit nectars

\begin{tabular}{|c|c|c|c|c|c|c|c|c|}
\hline $\begin{array}{c}\text { Sample } \\
\text { No. }\end{array}$ & Product & $\begin{array}{l}\text { Company } \\
\text { Name }\end{array}$ & $\begin{array}{l}\text { Volume of } \\
\text { Nectars }\end{array}$ & $\begin{array}{c}\text { Water } \\
\text { Capacity \% }\end{array}$ & $\mathrm{pH}$ Value & Acidity \% & TSS \% & $\begin{array}{c}\text { Artificial } \\
\text { Colors }\end{array}$ \\
\hline 1 & Grape & $\mathrm{E}$ & $990 \pm 10$ & $92.09 \pm 1.25$ & $3.93 \pm 0.12$ & $0.68 \pm 0.03$ & $14.8 \pm 0.5$ & -ve \\
\hline 2 & Grape & $\mathrm{L}$ & $980 \pm 10$ & $91.16 \pm 1.33$ & $4.91 \pm 0.10$ & $0.36 \pm 0.02$ & $14.4 \pm 0.6$ & -ve \\
\hline 3 & Pineapple & $\mathrm{E}$ & $990 \pm 10$ & $92.09 \pm 2.26$ & $4.01 \pm 0.08$ & $0.54 \pm 0.02$ & $6.9 \pm 0.2$ & -ve \\
\hline 4 & Pineapple & $\mathrm{F}$ & $980 \pm 7$ & $91.24 \pm 1.15$ & $3.90 \pm 0.13$ & $0.69 \pm 0.04$ & $6.6 \pm 0.2$ & -ve \\
\hline 5 & Pineapple & $\mathrm{L}$ & $980 \pm 8$ & $91.16 \pm 1.30$ & $4.00 \pm 0.15$ & $0.59 \pm 0.05$ & $9.9 \pm 0.3$ & -ve \\
\hline 6 & Pineapple & M & $980 \pm 10$ & $94.23 \pm 2.45$ & $3.69 \pm 0.20$ & $0.16 \pm 0.04$ & $13.5 \pm 0.4$ & -ve \\
\hline 7 & Pineapple & $\mathrm{N}$ & $980 \pm 8$ & $95.14 \pm 2.50$ & $3.48 \pm 0.10$ & $0.34 \pm 0.05$ & $13.6 \pm 0.5$ & -ve \\
\hline 8 & Mixed & $\mathrm{E}$ & $990 \pm 10$ & $92.09 \pm 2.33$ & $4.04 \pm 0.12$ & $0.48 \pm 0.04$ & $12.5 \pm 0.4$ & -ve \\
\hline 9 & Mixed & $\mathrm{F}$ & $980 \pm 10$ & $91.24 \pm 1.65$ & $4.97 \pm 0.10$ & $0.45 \pm 0.05$ & $6.4 \pm 0.3$ & -ve \\
\hline 10 & Mixed & $\mathrm{L}$ & $980 \pm 10$ & $91.16 \pm 1.44$ & $3.77 \pm 0.08$ & $0.37 \pm 0.06$ & $4.4 \pm 0.2$ & -ve \\
\hline 11 & Mixed & M & $980 \pm 10$ & $95.23 \pm 3.59$ & $3.90 \pm 0.10$ & $0.16 \pm 0.03$ & $14.2 \pm 0.5$ & -ve \\
\hline 12 & Mixed & $\mathrm{N}$ & $980 \pm 10$ & $95.14 \pm 2.81$ & $3.59 \pm 0.09$ & $0.13 \pm 0.04$ & $14.4 \pm 0.5$ & -ve \\
\hline 13 & Guava & M & $980 \pm 10$ & $95.23 \pm 2.90$ & $4.04 \pm 0.10$ & $0.20 \pm 0.05$ & $14.0 \pm 0.3$ & -ve \\
\hline 14 & Guava & $\mathrm{N}$ & $980 \pm 12$ & $95.14 \pm 4.75$ & $4.09 \pm 0.12$ & $0.41 \pm 0.07$ & $13.4 \pm 0.3$ & -ve \\
\hline 15 & Orange & $\mathrm{E}$ & $990 \pm 10$ & $92.09 \pm 2.82$ & $4.02 \pm 0.09$ & $0.69 \pm 0.05$ & $5.6 \pm 0.2$ & -ve \\
\hline 16 & Orange & $\mathrm{F}$ & $980 \pm 8$ & $91.24 \pm 1.66$ & $4.01 \pm 0.10$ & $0.77 \pm 0.06$ & $6.5 \pm 0.2$ & -ve \\
\hline 17 & Orange & $\mathrm{L}$ & $980 \pm 5$ & $91.16 \pm 1.90$ & $4.61 \pm 0.15$ & $0.55 \pm 0.04$ & $9.9 \pm 0.3$ & -ve \\
\hline 18 & Orange & M & $980 \pm 7$ & $95.23 \pm 3.77$ & $3.80 \pm 0.08$ & $0.98 \pm 0.06$ & $15.7 \pm 0.5$ & -ve \\
\hline 19 & Orange & $\mathrm{N}$ & $980 \pm 10$ & $95.14 \pm 4.80$ & $3.27 \pm 0.09$ & $0.12 \pm 0.02$ & $14.5 \pm 0.4$ & -ve \\
\hline 20 & Apple & $\mathrm{E}$ & $990 \pm 10$ & $92.09 \pm 1.75$ & $3.73 \pm 0.10$ & $0.41 \pm 0.05$ & $6.0 \pm 0.2$ & -ve \\
\hline 21 & Apple & $\mathrm{F}$ & $980 \pm 10$ & $91.24 \pm 1.95$ & $3.86 \pm 0.12$ & $0.53 \pm 0.04$ & $5.9 \pm 0.2$ & -ve \\
\hline 22 & Apple & M & $980 \pm 12$ & $95.23 \pm 3.89$ & $3.56 \pm 0.08$ & $0.28 \pm 0.03$ & $15.2 \pm 0.4$ & -ve \\
\hline 23 & Apple & $\mathrm{N}$ & $980 \pm 10$ & $95.14 \pm 3.71$ & $3.75 \pm 0.10$ & $0.39 \pm 0.05$ & $14.0 \pm 0.5$ & -ve \\
\hline
\end{tabular}

Table 3 Heavy metals $(\mathrm{mg} / \mathrm{kg}$ ) of local produced fruit nectars

\begin{tabular}{c|cccccccc}
\hline $\begin{array}{c}\text { Sample } \\
\text { No. }\end{array}$ & Product & $\begin{array}{c}\text { Company } \\
\text { Name }\end{array}$ & $\begin{array}{c}\mathrm{Fe} \\
\mathrm{mg} / \mathrm{kg}\end{array}$ & $\begin{array}{c}\mathrm{Zn} \\
\mathrm{mg} / \mathrm{kg}\end{array}$ & $\begin{array}{c}\mathrm{Cu} \\
\mathrm{mg} / \mathrm{kg}\end{array}$ & $\begin{array}{c}\mathrm{Ar} \\
\mathrm{mg} / \mathrm{kg}\end{array}$ & $\begin{array}{c}\mathrm{Pb} \\
\mathrm{mg} / \mathrm{kg}\end{array}$ & $\begin{array}{c}\mathrm{Cd} \\
\mathrm{mg} / \mathrm{kg}\end{array}$ \\
\hline 1 & Grape & $\mathrm{B}$ & $5.75 \pm 1.23$ & $2.23 \pm 0.35$ & $3.02 \pm 0.50$ & $0.04 \pm 0.01$ & $0.008 \pm 0.001$ & $0.006 \pm 0.002$ \\
2 & Grape & $\mathrm{C}$ & $5.90 \pm 0.89$ & $3.22 \pm 0.55$ & $2.73 \pm 0.47$ & $0.06 \pm 0.02$ & $0.005 \pm 0.002$ & $0.003 \pm 0.001$ \\
3 & Grape & $\mathrm{D}$ & $7.30 \pm 1.23$ & $1.87 \pm 0.23$ & $1.66 \pm 0.75$ & $0.02 \pm 0.005$ & $0.003 \pm 0.001$ & $0.005 \pm 0.002$ \\
4 & Pineapple & $\mathrm{A}$ & $4.45 \pm 0.98$ & $2.61 \pm 0.19$ & $2.85 \pm 0.92$ & $0.04 \pm 0.01$ & $0.005 \pm 0.002$ & $0.002 \pm 0.001$ \\
5 & Pineapple & $\mathrm{B}$ & $6.15 \pm 0.45$ & $2.50 \pm 0.37$ & $1.99 \pm 0.89$ & $0.02 \pm 0.006$ & $0.006 \pm 0.003$ & $0.007 \pm 0.003$ \\
6 & Pineapple & $\mathrm{C}$ & $5.87 \pm 0.95$ & $3.52 \pm 0.61$ & $2.81 \pm 0.48$ & $0.02 \pm 0.008$ & $0.006 \pm 0.002$ & $0.005 \pm 0.002$ \\
7 & Pineapple & $\mathrm{D}$ & $6.32 \pm 0.85$ & $2.75 \pm 0.39$ & $1.56 \pm 0.76$ & $0.03 \pm 0.01$ & $0.002 \pm 0.001$ & $0.008 \pm 0.004$ \\
8 & Mixed & $\mathrm{A}$ & $6.34 \pm 1.05$ & $3.04 \pm 0.22$ & $1.76 \pm 0.73$ & $0.05 \pm 0.003$ & $0.002 \pm 0.001$ & $0.008 \pm 0.003$ \\
9 & Mixed & $\mathrm{C}$ & $6.76 \pm 0.87$ & $2.18 \pm 0.41$ & $2.97 \pm 0.91$ & $0.04 \pm 0.01$ & $0.006 \pm 0.002$ & $0.003 \pm 0.001$ \\
10 & Guava & $\mathrm{A}$ & $5.90 \pm 0.75$ & $2.71 \pm 0.33$ & $2.28 \pm 0.85$ & $0.07 \pm 0.01$ & $0.003 \pm 0.001$ & $0.004 \pm 0.002$ \\
11 & Guava & $\mathrm{B}$ & $7.10 \pm 1.09$ & $3.02 \pm 0.65$ & $2.08 \pm 0.75$ & $0.03 \pm 0.007$ & $0.006 \pm 0.002$ & $0.008 \pm 0.005$ \\
12 & Guava & $\mathrm{C}$ & $5.76 \pm 0.83$ & $3.06 \pm 0.47$ & $1.86 \pm 0.25$ & $0.03 \pm 0.005$ & $0.005 \pm 0.002$ & $0.006 \pm 0.002$ \\
13 & Orange & $\mathrm{A}$ & $7.23 \pm 0.95$ & $2.90 \pm 0.18$ & $3.23 \pm 0.54$ & $0.02 \pm 0.008$ & $0.007 \pm 0.003$ & $0.006 \pm 0.003$ \\
14 & Orange & $\mathrm{B}$ & $6.89 \pm 0.72$ & $4.01 \pm 0.68$ & $2.45 \pm 0.51$ & $0.05 \pm 0.009$ & $0.003 \pm 0.001$ & $0.004 \pm 0.001$ \\
15 & Apple & $\mathrm{A}$ & $6.79 \pm 0.91$ & $2.76 \pm 0.34$ & $3.20 \pm 0.63$ & $0.06 \pm 0.01$ & $0.004 \pm 0.001$ & $0.006 \pm 0.002$ \\
16 & Apple & $\mathrm{C}$ & $7.22 \pm 1.03$ & $2.90 \pm 0.29$ & $3.05 \pm 0.42$ & $0.05 \pm 0.01$ & $0.003 \pm 0.001$ & $0.004 \pm 0.001$ \\
17 & Apple & D & $4.45 \pm 0.76$ & $1.77 \pm 0.37$ & $2.31 \pm 0.22$ & $0.04 \pm 0.008$ & $0.004 \pm 0.002$ & $0.006 \pm 0.003$ \\
\hline
\end{tabular}

\section{Heavy Metals}

The quantity of heavy metals for all local fruit nectar samples were within the permissible level of the Libyan specification standard (990-2014) for fruit nectars, which stated that heavy metals (iron, zinc, copper, arsenic, lead, cadmium) should be no more than $(15,5,5,0.1,0.01$, $0.01) \mathrm{mg} / \mathrm{kg}$, respectively, the concentration of $\mathrm{Fe}$ was ranged between (7.30-4.45), $\mathrm{Zn}$ (4.01-1.77), $\mathrm{Cu}$ (3.231.56), $\mathrm{Ar}(0.07-0.02), \mathrm{Pb}(0.008-0.002)$ and $\mathrm{Cd}(0.008-$ $0.002) \mathrm{mg} / \mathrm{kg}$ (Table 3).
However, arsenic contents in some of the imported fruit nectars samples (6 pineapple, 12 mixed, 13 guava) were $(0.18,0.15,0.16) \mathrm{mg} / \mathrm{kg}$ which produced by $(\mathrm{m}, \mathrm{n}$, $\mathrm{m})$ companies respectively were unconfirmed with the Libyan specification standard for fruit nectars which indicated that arsenic should be not more than $(0.1$ $\mathrm{mg} / \mathrm{kg}$ ), the concentration of $\mathrm{Fe}$ was ranged between (9.02-3.56), Zn (4.05-1.85), Cu (4.54-1.75), Ar (0.18$0.01), \mathrm{Pb}(0.006-0.002)$ and $\mathrm{Cd}(0.008-0.002) \mathrm{mg} / \mathrm{kg}$ (Table 4). 
Table 4 Heavy metals $(\mathrm{mg} / \mathrm{kg})$ of imported fruit nectars

\begin{tabular}{|c|c|c|c|c|c|c|c|c|}
\hline $\begin{array}{c}\text { Sample } \\
\text { No. }\end{array}$ & Product & $\begin{array}{l}\text { Company } \\
\text { Name }\end{array}$ & $\begin{array}{c}\mathrm{Fe} \\
\mathrm{mg} / \mathrm{kg}\end{array}$ & $\begin{array}{c}\mathrm{Zn} \\
\mathrm{mg} / \mathrm{kg}\end{array}$ & $\begin{array}{c}\mathrm{Cu} \\
\mathrm{mg} / \mathrm{kg}\end{array}$ & $\begin{array}{c}\mathrm{Ar} \\
\mathrm{mg} / \mathrm{kg}\end{array}$ & $\begin{array}{c}\mathrm{Pb} \\
\mathrm{mg} / \mathrm{kg}\end{array}$ & $\begin{array}{c}\mathrm{Cd} \\
\mathrm{mg} / \mathrm{kg}\end{array}$ \\
\hline 1 & Grape & E & $8.08 \pm 1.08$ & $1.87 \pm 0.55$ & $3.98 \pm 0.64$ & $0.02 \pm 0.001$ & $0.005 \pm 0.002$ & $0.003 \pm 0.001$ \\
\hline 2 & Grape & $\mathrm{L}$ & $5.02 \pm 0.93$ & $4.03 \pm 0.46$ & $1.75 \pm 0.81$ & $0.05 \pm 0.004$ & $0.002 \pm 0.001$ & $0.003 \pm 0.002$ \\
\hline 3 & Pineapple & E & $5.59 \pm 0.65$ & $4.05 \pm 0.73$ & $2.65 \pm 0.69$ & $0.06 \pm 0.003$ & $0.002 \pm 0.001$ & $0.005 \pm 0.002$ \\
\hline 4 & Pineapple & $\mathrm{F}$ & $7.54 \pm 0.72$ & $2.74 \pm 0.53$ & $3.34 \pm 0.28$ & $0.03 \pm 0.007$ & $0.005 \pm 0.002$ & $0.008 \pm 0.004$ \\
\hline 5 & Pineapple & $\mathrm{L}$ & $6.91 \pm 0.81$ & $2.77 \pm 0.46$ & $2.53 \pm 0.73$ & $0.01 \pm 0.005$ & $0.004 \pm 0.002$ & $0.007 \pm 0.003$ \\
\hline 6 & Pineapple & M & $4.16 \pm 0.74$ & $3.65 \pm 0.61$ & $2.54 \pm 0.43$ & $0.18 \pm 0.04$ & $0.002 \pm 0.001$ & $0.002 \pm 0.001$ \\
\hline 7 & Pineapple & $\mathrm{N}$ & $5.91 \pm 0.84$ & $4.02 \pm 0.38$ & $2.94 \pm 0.62$ & $0.08 \pm 0.009$ & $0.006 \pm 0.002$ & $0.008 \pm 0.004$ \\
\hline 8 & Mixed & E & $4.45 \pm 0.55$ & $3.34 \pm 0.29$ & $2.76 \pm 0.51$ & $0.04 \pm 0.005$ & $0.003 \pm 0.001$ & $0.002 \pm 0.001$ \\
\hline 9 & Mixed & $\mathrm{F}$ & $6.19 \pm 0.36$ & $2.98 \pm 0.32$ & $4.54 \pm 0.33$ & $0.10 \pm 0.03$ & $0.004 \pm 0.001$ & $0.006 \pm 0.003$ \\
\hline 10 & Mixed & $\mathrm{L}$ & $5.15 \pm 0.54$ & $2.81 \pm 0.15$ & $1.88 \pm 0.49$ & $0.06 \pm 0.004$ & $0.003 \pm 0.001$ & $0.002 \pm 0.001$ \\
\hline 11 & Mixed & M & $8.30 \pm 1.03$ & $3.42 \pm 0.18$ & $2.51 \pm 0.23$ & $0.05 \pm 0.003$ & $0.006 \pm 0.001$ & $0.008 \pm 0.005$ \\
\hline 12 & Mixed & $\mathrm{N}$ & $3.56 \pm 0.78$ & $2.34 \pm 0.23$ & $1.99 \pm 0.28$ & $0.15 \pm 0.06$ & $0.005 \pm 0.001$ & $0.006 \pm 0.003$ \\
\hline 13 & Guava & M & $9.02 \pm 1.05$ & $2.75 \pm 0.32$ & $1.76 \pm 0.19$ & $0.16 \pm 0.03$ & $0.003 \pm 0.001$ & $0.002 \pm 0.001$ \\
\hline 14 & Guava & $\mathrm{N}$ & $7.02 \pm 0.48$ & $3.55 \pm 0.19$ & $2.65 \pm 0.45$ & $0.05 \pm 0.007$ & $0.003 \pm 0.001$ & $0.004 \pm 0.002$ \\
\hline 15 & Orange & E & $6.85 \pm 0.59$ & $3.08 \pm 0.20$ & $2.19 \pm 0.30$ & $0.04 \pm 0.005$ & $0.004 \pm 0.001$ & $0.005 \pm 0.003$ \\
\hline 16 & Orange & $\mathrm{F}$ & $5.41 \pm 0.62$ & $3.56 \pm 0.48$ & $1.76 \pm 0.29$ & $0.06 \pm 0.008$ & $0.002 \pm 0.001$ & $0.007 \pm 0.002$ \\
\hline 17 & Orange & $\mathrm{L}$ & $6.82 \pm 0.49$ & $3.45 \pm 0.62$ & $2.27 \pm 0.61$ & $0.03 \pm 0.007$ & $0.005 \pm 0.002$ & $0.003 \pm 0.001$ \\
\hline 18 & Orange & M & $4.74 \pm 0.28$ & $1.85 \pm 0.16$ & $2.82 \pm 0.48$ & $0.07 \pm 0.005$ & $0.004 \pm 0.001$ & $0.006 \pm 0.002$ \\
\hline 19 & Orange & $\mathrm{N}$ & $6.80 \pm 0.75$ & $2.88 \pm 0.45$ & $3.71 \pm 0.29$ & $0.04 \pm 0.003$ & $0.004 \pm 0.001$ & $0.007 \pm 0.003$ \\
\hline 20 & Apple & E & $7.24 \pm 0.92$ & $2.16 \pm 0.29$ & $3.05 \pm 0.42$ & $0.07 \pm 0.008$ & $0.006 \pm 0.002$ & $0.003 \pm 0.001$ \\
\hline 21 & Apple & $\mathrm{F}$ & $6.10 \pm 0.84$ & $2.31 \pm 0.28$ & $2.56 \pm 0.30$ & $0.04 \pm 0.007$ & $0.003 \pm 0.001$ & $0.005 \pm 0.002$ \\
\hline 22 & Apple & M & $6.70 \pm 0.66$ & $1.90 \pm 0.17$ & $3.17 \pm 0.46$ & $0.06 \pm 0.005$ & $0.003 \pm 0.001$ & $0.004 \pm 0.002$ \\
\hline 23 & Apple & $\mathrm{N}$ & $6.84 \pm 0.51$ & $2.72 \pm 0.35$ & $4.12 \pm 0.72$ & $0.06 \pm 0.004$ & $0.002 \pm 0.001$ & $0.005 \pm 0.001$ \\
\hline
\end{tabular}

Table 5 Microbiological results of local produced fruit nectars

\begin{tabular}{|c|c|c|c|c|c|}
\hline $\begin{array}{l}\text { Sample } \\
\text { No. }\end{array}$ & Product & $\begin{array}{c}\text { Company } \\
\text { Name }\end{array}$ & $\begin{array}{c}\text { Total Bacterial Count } \\
(\mathrm{cfu} / \mathrm{ml})\end{array}$ & Yeasts \&Molds (cfu/ml) & $\begin{array}{c}\text { Coliform Bacteria } \\
(\mathrm{cfu} / \mathrm{ml})\end{array}$ \\
\hline 1 & Grape & $\mathrm{B}$ & - ve & -ve & $-\mathrm{ve}$ \\
\hline 2 & Grape & $\mathrm{C}$ & -ve & -ve & -ve \\
\hline 3 & Grape & $\mathrm{D}$ & -ve & -ve & -ve \\
\hline 4 & Pineapple & A & -ve & -ve & -ve \\
\hline 5 & Pineapple & B & -ve & -ve & -ve \\
\hline 6 & Pineapple & $\mathrm{C}$ & -ve & -ve & -ve \\
\hline 7 & Pineapple & $\mathrm{D}$ & -ve & -ve & -ve \\
\hline 8 & Mixed & A & -ve & -ve & -ve \\
\hline 9 & Mixed & $\mathrm{C}$ & -ve & -ve & -ve \\
\hline 10 & Guava & A & -ve & -ve & -ve \\
\hline 11 & Guava & $\mathrm{B}$ & -ve & -ve & -ve \\
\hline 12 & Guava & $\mathrm{C}$ & -ve & -ve & -ve \\
\hline 13 & Orange & A & -ve & -ve & -ve \\
\hline 14 & Orange & B & -ve & -ve & -ve \\
\hline 15 & Apple & A & -ve & -ve & -ve \\
\hline 16 & Apple & $\mathrm{C}$ & -ve & -ve & -ve \\
\hline 17 & Apple & $\mathrm{D}$ & -ve & -ve & -ve \\
\hline
\end{tabular}

\section{Microbiological Analysis}

Microbiological analysis results in table (5\&.6) indicated that the local and imported fruit nectars samples were free from total coliform bacteria, bacterial count, yeasts and molds which matching the Libyan specification standard (990-2014).

\section{Questionnaire Results}

Throughout the questionnaire prepared as a part of this study indicated after graphically and statistically analyzing, that the average consumption of the individual Libyan consumer was about 36.5 liters/year/capita, also the questionnaire indicated that $25 \%$ of the consumers usually consume 1 cup a day of fruit nectars or juices, 
$35 \%$ of consumers may consume 2 cups/day and only $10 \%$ consume more than 3 cup/day (Figure 1).The more brands consumed by different age groups of consumers were the imported brands $(50 \%)$, and local brands accounted as $(33 \%)$ and both of them together were $(17 \%)$ (Figure 2). Also the results showed that $83 \%$ of Libyan consumers most of the time consume fruit juices and nectars and $13 \%$ did not consumed them.

Table 6 Microbiological results of imported fruit nectars

\begin{tabular}{|c|c|c|c|c|c|}
\hline $\begin{array}{l}\text { Sample } \\
\text { No. }\end{array}$ & Product & $\begin{array}{l}\text { Company } \\
\text { Name }\end{array}$ & $\begin{array}{l}\text { Total Bacterial Count } \\
(\mathrm{cfu} / \mathrm{ml})\end{array}$ & Yeasts \& Molds (cfu/ml) & $\begin{array}{l}\text { Coliform Bacteria } \\
\text { (cfu/ml) }\end{array}$ \\
\hline 1 & Grape & $\mathrm{E}$ & - ve & -ve & -ve \\
\hline 2 & Grape & $\mathrm{L}$ & -ve & -ve & -ve \\
\hline 3 & Pineapple & $\mathrm{E}$ & -ve & -ve & -ve \\
\hline 4 & Pineapple & $\mathrm{F}$ & -ve & -ve & -ve \\
\hline 5 & Pineapple & $\mathrm{L}$ & -ve & -ve & -ve \\
\hline 6 & Pineapple & M & -ve & -ve & -ve \\
\hline 7 & Pineapple & $\mathrm{N}$ & -ve & -ve & -ve \\
\hline 8 & Mixed & $\mathrm{E}$ & -ve & -ve & -ve \\
\hline 9 & Mixed & $\mathrm{F}$ & -ve & -ve & -ve \\
\hline 10 & Mixed & $\mathrm{L}$ & -ve & -ve & -ve \\
\hline 11 & Mixed & M & -ve & -ve & -ve \\
\hline 12 & Mixed & $\mathrm{N}$ & -ve & -ve & -ve \\
\hline 13 & Guava & M & -ve & -ve & -ve \\
\hline 14 & Guava & $\mathrm{N}$ & -ve & -ve & -ve \\
\hline 15 & Orange & $\mathrm{E}$ & -ve & -ve & -ve \\
\hline 16 & Orange & $\mathrm{F}$ & -ve & -ve & -ve \\
\hline 17 & Orange & $\mathrm{L}$ & -ve & -ve & -ve \\
\hline 18 & Orange & M & -ve & -ve & -ve \\
\hline 19 & Orange & $\mathrm{N}$ & -ve & -ve & -ve \\
\hline 20 & Apple & $\mathrm{E}$ & -ve & -ve & -ve \\
\hline 21 & Apple & $\mathrm{F}$ & -ve & -ve & -ve \\
\hline 22 & Apple & M & -ve & -ve & -ve \\
\hline 23 & Apple & $\mathrm{N}$ & -ve & -ve & -ve \\
\hline
\end{tabular}

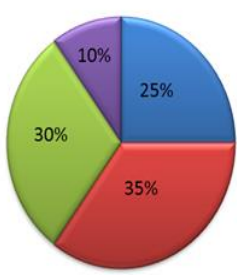

Figure 1 Daily rate consumption of fruit juices and nectars (cup/daily/capita)

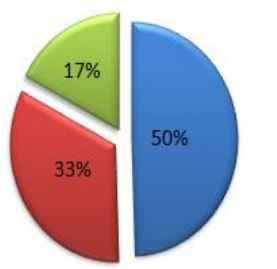

Figure 2 Local and imported trademarks of fruit nectars preferred by consumer

\section{Discussion}

Finding results of this study indicated that some local and imported fruit nectar samples were unconfirmed with the Libyan specification standard, such as titratable acidity were high in some samples which could be as a result of immaturity of the fruits used in the processing of nectars. All samples were indicated that free of artificial colors which could be added to fruit nectars or juices, addition of artificial colors to fruit nectars and juices contrary to Libyan and international legislation and specification standards, as a result of the seriousness of these artificial colors on the health and consumer safety. With regard to the percentage of TSS, the results of this study were consistent with a study of (Maireva et al., 2013), where found decrease in the percentage of TSS in $(19.5 \%)$ of different fruit nectars samples which were below the limits of the specification standard.

Finding results were consistent with the study conducted by (Nascentes et al., 2004) in Brazil for determination of copper and zinc in fruit juices, they found variation in the concentration of copper and zinc 
but within the limits of the approved specifications (Nascentes et al., 2004).The results of this study were incompatible with the research conducted by (Rashed et al., 2013) for microbiological analyzes of some fruit juice samples sold in Bangladesh local markets which indicated that total bacteria count was between $10^{2}-10^{7}(\mathrm{cfu} / \mathrm{ml})$ and number of coliform bacteria ranged between $10^{2}-10^{6}$ (cfu/ml), thus these samples were unconfirmed with the standard specifications of the juices approved in Bangladesh (Rashed et al., 2013).

The questionnaire results illustrated that average consumption of the Libyan consumer was about 36.5 liters/year/capita which was compatible with some of other Arabic countries, such as Saudi Arabia, UAE and Kuwait (Free Palestine Agency, 2014).

\section{Conclusion}

Through the results of this study which conducted physical, chemical, microbiological and heavy metals tests for local and imported fruit nectar samples, indicated that some samples does not meet the Libyan specification standard of fruit nectars as follows:

- High pH values for 5 local produced samples and 4 imported samples.

- High acidity of one local produced sample and 5 imported samples.

- Low total soluble solids in 6 local produced samples and 10 imported samples were accounted $35.3 \%$ and $43.5 \%$ of the total number of local produced and imported sample respectively.

- All locally produced and imported samples were free of coliform bacteria, total bacterial count, yeasts and molds.

- High quantity of arsenic for 3 imported samples.

- Based on these results we recommend the following:

- Emphasis on the importance of conducting analyzes and tests for various locally and imported produced foods, including fruits nectar not only during production but also during handling operations, storage and marketing by the regulatory authorities.

- Conducting studies and scientific technical research for the quality control of different fruit nectars as well as other food products for the sake of health and safety of the consumer.

- Emphasize the role of the National Center for specifications and standards to renewing and the development of Libyan specification standard for fruit juices and other food standards.

\section{References}

AOAC. 1984. Association of Official Analytical Chemists. Heavy metals analysis. Method. No. 25.061.

ASSABAH. 2014. www.assabah.press.ma.com, Fruit nectars and nutritive value (Accessed date 8-2014)

Codex Alimentarius. 2005. General standard for fruit juice and nectar. Codex Standard 247-2005.

Free Palestine Agency, 2014. Consumption rate of fruit juices of some Arabic countries. www.palestineafree.com (Accessed date 8-2014)

IFU. 1996a. International Federation of Fruit Juice Producers. Total count of potential spoilaging microorganisms of fruit and related products. No. 2 .

IFU. 1996b. International Federation of Fruit Juice Producers. Yeast and moulds count procedure. No. $3 \& 4$.

IFU. 2005. International Federation of Fruit Juice Producer. Detection of water soluble artificial colorants. No.24.

ISO. 1991. International Organization of Standards. Fruit and vegetable products-determination of pH. No. 1842.

ISO. 1998a. International Organization of Standards. Fruit and vegetable products-determination of soluble solidsrefractometric method. No. 2173.

ISO. 1998b. International Organization of Standards. Fruit and vegetable products-determination of titratable acidity. No. 750 .

ISO. 2006. International Organization of Standards. Fruit and vegetable products-detection of coliform bacteria. No. 4832$1 \& 2$.

Leahu A, Damian C, Oroian M, Ropciuc S. 2013. Physical-chemical parameters of fruit juices-evolution during storage. Lucrari Stiintifice-Seria Zootehnie. 59: 213-217

Maireva S; Usai T, Manhokwe S. 2013. The determination of adulteration in orange based fruit juices. International Journal of Science and Technology. 2(5): 365-372.

Nascentes C, Arruda Z, Noguerira R, Nobrega JA. 2004 Direct determination of $\mathrm{Cu}$ and $\mathrm{Zn}$ in fruit juices and bovine milk by theromospray flame furnace atomic absorption spectrometry. Talanta.64: 912-917.

NCSM. 2014. National Center of Standards and Meteorology, Libyan specification standards for fruit nectars, (990-2014), Tripoli, Libya.

Ofori H, Owusu M, Anyebuno G. 2013. Heavy metal analysis of fruit juice and soft drinks bought from retail market in Accra Ghana. Journal of Scientific Research and Reports. 2(1): 423428.

Rashed N, Aftab U, Azizul H. 2013. Microbiological study of vendor and packed fruit juices locally available in Dhaka city, Bangladesh. International Food Research Journal. 20 (2): 10111015 . 